\title{
The early variation of left ventricular twisting function in patients with lymphoma received anthracycline therapy assessed by three-dimensional speckle tracking echocardiography
}

\author{
Feiyan Song ${ }^{1 \#}$, Yu Kang ${ }^{2 \#}$, Chujie Zhang ${ }^{1}$, Yuchen $\mathrm{Xu}^{1}$, Jing $\mathrm{Shi}^{1}$, Ye Guo ${ }^{3}$, \\ Qunling Zhang ${ }^{3}$, Xianhong Shu ${ }^{1}$, Leilei Cheng ${ }^{1}$
}

${ }^{1}$ Department of Echocardiography, Zhongshan Hospital, Fudan University,

Shanghai Institute of Cardiovascular Diseases, Shanghai Institute of Medical Imaging, Shanghai, China

${ }^{2}$ Department of Cardiology, Renji Hospital, School of Medicine,

Shanghai Jiaotong University, Shanghai, China

${ }^{3}$ Department of Medical Oncology, Fudan University Shanghai Cancer Center, Shanghai, China

\begin{abstract}
Background: Anthracycline-induced cardiotoxicity remains a significant and unresolved issue in patients receiving chemotherapy. The aim of this study was to evaluate left ventricular (LV) twisting function by three-dimensional speckle tracking echocardiography (3D-STE) in patients with lymphoma after anthracycline therapy.

Methods: One hundred and one patients with newly diagnosed diffuse large B-cell lymphoma who had planned to receive anthracycline chemotherapy were enrolled. LV apical rotation, basal rotation, twist, torsion, time to peak apical rotation and time to peak basal rotation were measured by $3 D-S T E$ at baseline, after the completion of two cycles and four cycles of the regimen, respectively. Apical-basal rotation delay was calculated as the difference between time to basal and time to apical rotation.

Results: The results showed that $L V$ apical rotation, basal rotation, twist and torsion declined progressively during the whole procedure (baseline vs. two and four cycles of the regimen, apical rotation: $12.5 \pm$ $\pm 4.5^{\circ}$ vs. $8.8 \pm 3.6^{\circ}$ vs. $6.0 \pm 3.2^{\circ}$; basal rotation: $-7.7 \pm 3.0^{\circ}$ vs. $-5.9 \pm 2.6^{\circ}$ vs. $-4.4 \pm 2.5^{\circ}$; twist: $20.0 \pm 6.4^{\circ}$ vs. $14.5 \pm 5.1^{\circ}$ vs. $9.8 \pm 4.5^{\circ}$; torsion: $2.9 \pm 0.9^{\circ} / \mathrm{cm}$ vs. $2.1 \pm 0.9^{\circ} / \mathrm{cm}$ vs. $1.4 \pm 0.7^{\circ} / \mathrm{cm}$; all $p<0.01)$. Furthermore, apical-basal rotation delay increased significantly after two cycles as well as after four cycles of the regimen ( $38.3 \pm 67.9 \mathrm{~ms} v$ s. $66.7 \pm 73.9 \mathrm{~ms} v \mathrm{~s} .92 .6 \pm 96.9 \mathrm{~ms} ; p<0.01)$. Conclusions: $L V$ twisting function deteriorated in the early stage of anthracycline therapy in patients with lymphoma, which could be detected by 3D-STE sensitively. (Cardiol J 2017; 24, 5: 484-494)

Key words: anthracycline, cardiac toxicity, left ventricular function, twist, echocardiography
\end{abstract}

Address for correspondence:

Leilei Cheng, MD, PhD, Department of Echocardiography, Zhongshan Hospital, Fudan University, Shanghai Institute of Cardiovascular Diseases, Shanghai Institute of Medical Imaging, 180 Fenglin Road, Shanghai, China, tel: +8602164038038, e-mail: cheng.leilei@zs-hospital.sh.cn

Xianhong Shu, MD, PhD, Department of Echocardiography, Zhongshan Hospital, Fudan University, Shanghai Institute of Cardiovascular Diseases, Shanghai Institute of Medical Imaging, 180 Fenglin Road, Shanghai, China, tel: +86 021 64223006, e-mail: shu.xianhong@zs-hospital.sh.cn

${ }^{\#}$ These authors contributed equally to this study and should be considered co-first authors.

Received: 14.12.2016 Accepted: 24.02.2017 


\section{Introduction}

At present, anthracycline continues to be a cornerstone in the treatment of lymphoma, although the established risk of cardiac side effects may limit its use due to concerns about patient prognosis [1]. Previous studies have suggested that anthracycline-induced cardiotoxicity had emerged as the leading cause of noncancerous morbidity and mortality in patients exposed to anthracycline therapy $[2,3]$. Left ventricular (LV) dysfunction is the most common and serious side effect of cancer treatment. Several studies have reported LV twisting behavior was sensitive to detect abnormalities of $\mathrm{LV}$ function in various conditions of cardiovascular diseases (CVD) [4-8]. $\mathrm{LV}$ twisting is an important deformation for cardiac motion in a three-dimensional space (3D), however, accurate quantification of LV twisting function remains challenging. Although cardiac magnetic resonance (CMR) imaging is the gold standard to measure myocardial mechanics in the setting of a wide variety of myocardial disease processes, it is constrained by its availability and cost as a first-line technique for serial evaluation of LV dysfunction in clinical practice. Recently, introduced 3D speckle tracking echocardiography (3D-STE) has proved to be feasible and comparable to CMR imaging in the assessment of LV rotation function [9]. Thus, the aim of this study was to examine LV twisting by $3 \mathrm{D}-\mathrm{STE}$ in patients with lymphoma exposed to anthracycline therapy.

\section{Methods}

\section{Study subjects}

A total of 101 patients with newly diagnosed and histopathologically confirmed diffuse large B-cell lymphoma between December 2012 and August 2015 at Fudan University Shanghai Cancer Center were enrolled prospectively and received anthracycline-embedded chemotherapy. The mean age of these patients was 49 years, with a range of 20-78 years. All patients were treated with 4 cycles of R-CHOP (cyclophosphamide $750 \mathrm{mg} / \mathrm{m}^{2}$, vincristine $1.4 \mathrm{mg} / \mathrm{m}^{2}$ up to a maximum dose of $2 \mathrm{mg} / \mathrm{m}^{2}$, epirubicin $50 \mathrm{mg} / \mathrm{m}^{2}$ on day 1 , prednisone $100 \mathrm{mg}$ on days $1-5$, and rituximab $375 \mathrm{mg} / \mathrm{m}^{2}$ ). Patients were restaged every 2 cycles, and those with disease progression were withdrawn from the treatment of R-CHOP. None of the patients received other cardiotoxic agents, radiation therapy, or cardiac protective protocols during the entire study. Patients' age $<18$ years, viral myocarditis, severe hypertension, life expectancy $\leq 12$ weeks, serious arrhythmia, renal or hepatic dysfunction, respiratory failure, valvular heart disease, a previous history of heart failure and/or coronary artery disease were exclusion criteria for enrollment. All subjects provided informed consent for participation in this study and for the administration of anthracycline therapy. The local ethics committee approved the protocol (The ethics approval number of Fudan University Shanghai Cancer Center is: 1212117-6, The ethics approval number of Zhongshan Hospital of Fudan University is: 2011-117).

\section{Echocardiographic imaging}

All subjects underwent traditional two-dimensional (2D) echocardiography and 3D-STE examination at baseline, 1 day after the completion of 2 cycles and 4 cycles of the regimen. Images were obtained with a commercially available ultrasound machine (iE33, Philips Medical Systems, Andover WA, USA) equipped with S5-1 (1 to $5 \mathrm{MHz}$ ) and X3-1 (1 to $3 \mathrm{MHz}$ ) transducer. Standard 2D and 3D echocardiography were performed according to the recommendations of American Society of Echocardiography [10]. Five consecutive cardiac cycle for 2D images and 6 consecutive cardiac cycle for 3D-STE images were acquired. The image parameters such as depth, sector size, angle and focus were optimized to achieve the frame rate with a range of 60-80 FPS (frames per second) for 2D and 30-45 FPS for 3D-STE analysis. At least three apical 4-chamber 3D-STE images for each patient were digitally stored for offline analysis. Echocardiography technicians as well as off-line echo readers were blinded to the clinical data and biochemical results.

\section{Diastolic function measurements}

Pulsed-wave (PW) Doppler variables (mitral $\mathrm{E}$ and $\mathrm{A}$ diastolic waves, $\mathrm{E}$ wave deceleration time [DT], isovolumetric relaxation time [IVRT]) and tissue Doppler data imaging (TDI) (mitral annular diastolic E' and A' waves) were obtained according to standard guidelines [10].

\section{Offline analysis of 3D-STE}

TomTec 4D LV analysis (4.6.0.411, TomTec Imaging Systems GMBH, Germany) was used for 3D-STE data analysis. It was performed by making two reference points at the middle of mitral valve annulus and the apex respectively in three longaxis reference planes (apical 4-chamber, 2-chamber, and 3-chamber views) and orientating one reference point at the annulus aortic valve in short-axis 
reference plane. LV end-diastolic volume (EDV), end-systolic volume (ESV), stroke volume, ejection fraction (EF), and global longitudinal strain (GLS) as well as LV mass were automatically calculated. As a marker of global LV dyssynchrony, the systolic dyssynchrony index (SDI) was defined as the standard deviation of time taken to reach the minimal regional volume for each of the 16 segments as a percentage of the cardiac cycle. Rotation of LV is the wringing motion of the ventricle around its long axis. The base rotates in an overall clockwise direction and the apex rotates in a counterclockwise direction when viewed from apex to base. Basal and apical rotation was determined by software after analysis. LV twist was defined as the difference in rotation between base and apex. The LV torsion was calculated as twist divided by LV length which was the distance between the middle of mitral valve annulus and the apex. To evaluate synchronicity between basal and apical rotation, the interval from the beginning of the QRS complex on the electrocardiogram to peak basal rotation (time to peak basal rotation) and the interval from the beginning of the QRS complex on the electrocardiogram to peak apical rotation (time to peak apical rotation) were obtained. Apical-basal rotation delay was calculated as the difference between time to basal and time to apical rotation. Manual correction was performed to optimize the endocardial border delineation in all patients.

\section{Assays for serum biochemical markers}

Serial serum samples were also collected at baseline, 1 day after the second cycle of chemotherapy and the fourth cycle of the regimen. Measurements of high-sensitivity cardiac troponin T (hs-cTnT) were accomplished by the hs-cTnT one-step electrochemiluminescence immunoassay (Roche cobase 411) as previously reported [11]. All pro-B-type-natriuretic peptide (pro-BNP) analyses were performed using Elecsys pro-BNP II. Technologists recording the hs-c TnT and pro-BNP results were blinded to the participants who were responsible for clinical as well as echocardiographic data.

\section{Inter-observer and intra-observer variability}

Inter- and intra-observer reproducibility was assessed by calculating the difference between the values of 15 randomly selected patients measured by one observer twice and by a second observer.

\footnotetext{
Statistical analysis

Continuous variables were expressed as mean
} \pm standard deviation. The variables that were not normally distributed were logarithmically transformed before the analysis. The comparison of variables within each group versus the baseline was performed with one-way analysis of variance (ANOVA) followed by Bonferroni's test. Pearson's correlation analysis was used to assess for relationships between LV twisting parameters and EF, GLS, and hs-cTnT. Data were analyzed using standard statistical software (SPSS version 19.0; SPSS, Inc, Chicago, IL, USA). For all statistical evaluations of results, $\mathrm{p}$ values $<0.05$ were considered significant. Inter- and intra-observer reproducibility of twist and torsion were assessed using intraclass correlation coefficients (ICCs).

\section{Results}

\section{Study population}

A total of 160 patients were screened; 9 were excluded due to uncontrolled hypertension, coronary artery disease and viral myocarditis during follow-up; 22 were excluded from the analysis because of poor image quality which could not be recognized by the workstation; 28 patients were lost to follow-up. The remaining 101 patients, $60(59 \%)$ males, ranging from 20 to 78 years (mean age $49.3 \pm 12.2$ years) were included in the statistical analysis. After 2 cycles of chemotherapy, the cumulative dose of epirubicin in patients was $100 \mathrm{mg} / \mathrm{m}^{2}$. After 4 cycles of chemotherapy, the patient's cumulative dose of epirubicin was $200 \mathrm{mg} / \mathrm{m}^{2}$, which was not high dose. The demographics and characteristics of patients are shown in Table 1.

\section{Diastolic function assessments}

Early diastolic transmitral flow velocity (E), late diastolic transmitral flow velocity (A), DT as well as IVRT did not have significant difference between the pre- and post-therapies as well (all $\mathrm{p}>0.05)$. TDI measurements including early and late diastolic myocardial velocities (E' and A') also remained within the reference range in all patients (p > 0.05) (Table 2).

\section{D-STE}

Table 3 depicts various parameters obtained by 3D-STE. After 4 cycles of chemotherapy, LV stroke volume reduced from $44.7 \pm 9.9 \mathrm{~mL}$ to $40.0 \pm 11.4 \mathrm{~mL}$ and $\mathrm{EF}$ decreased from $62.3 \pm$ $\pm 5.3 \%$ to $58.2 \pm 6.2 \%$, GLS dropped from $-21.8 \pm$ $\pm 4.8 \%$ to $-19.3 \pm 2.9 \%$, while EDV, ESV as well as LV mass showed no obvious alteration. 
Table 1. Characteristics of lymphoma patients.

\begin{tabular}{lcccc}
\hline Variables & Baseline & Second cycle & Forth cycle & P \\
\hline Number & 101 & 101 & 101 & - \\
Male [\%] & $60(59 \%)$ & $60(59 \%)$ & $60(59 \%)$ & - \\
Age [years] & $49.3 \pm 12.2$ & $49.3 \pm 12.2$ & $49.3 \pm 12.2$ & 1.000 \\
Height [cm] & $167.1 \pm 6.5$ & $167.2 \pm 11.5$ & $166.8 \pm 6.6$ & 0.907 \\
Mass [kg] & $65.8 \pm 1.8$ & $65.4 \pm 11.5$ & $65.3 \pm 12.0$ & 0.948 \\
BSA [m $\left.{ }^{2}\right]$ & $1.7 \pm 0.2$ & $1.7 \pm 0.2$ & $1.7 \pm 0.2$ & 0.966 \\
HR [bpm] & $78.6 \pm 11.8$ & $80.0 \pm 11.5$ & $82.7 \pm 10.5$ & $0.034^{*}$ \\
SBP [mm Hg] & $115.6 \pm 8.5$ & $115.6 \pm 10.5$ & $113.1 \pm 9.4$ & 0.104 \\
DBP [mm Hg] & $75.5 \pm 7.6$ & $74.8 \pm 6.6$ & $74.2 \pm 7.3$ & 0.447 \\
\hline
\end{tabular}

${ }^{*} p<0.05$, compared between baselines and after the fourth cycle of therapy; BSA — body surface area; HR — heart rate; SBP — systolic blood pressure; DBP — diastolic blood pressure

Table 2. Diastolic functional assessment.

\begin{tabular}{lcccc}
\hline Variables & Baseline & Second cycle & Fourth cycle & P \\
\hline E wave [cm/s] & $71.51 \pm 17.7$ & $68.85 \pm 16.30$ & $66.64 \pm 15.27$ & 0.111 \\
A wave [cm/s] & $71.12 \pm 13.54$ & $68.51 \pm 15.76$ & $68.89 \pm 15.24$ & 0.459 \\
DT [ms] & $179.41 \pm 31.61$ & $183.71 \pm 34.61$ & $183.95 \pm 34.25$ & 0.557 \\
IVRT [ms] & $75.12 \pm 10.83$ & $72.45 \pm 9.93$ & $73.56 \pm 10.97$ & 0.199 \\
E' wave [cm/s] & $12.06 \pm 4.08$ & $12.06 \pm 4.08$ & $12.28 \pm 4.17$ & 0.843 \\
A' wave [cm/s] & $11.94 \pm 3.27$ & $12.02 \pm 3.24$ & $12.34 \pm 3.11$ & 0.642 \\
\hline
\end{tabular}

DT - deceleration time; IVRT — isovolumic relaxation time

Table 3. Three-dimensional speckle tracking echocardiography measurements.

\begin{tabular}{lcccc}
\hline Variables & Baseline & Second cycle & Fourth cycle & P \\
\hline End-diastolic volume [mL] & $72.4 \pm 15.8$ & $72.5 \pm 16.8$ & $71.0 \pm 17.9$ & 0.761 \\
End-systolic volume [mL] & $27.5 \pm 8.1$ & $30.2 \pm 9.1$ & $29.9 \pm 9.1$ & 0.056 \\
Stroke volume [mL] & $44.7 \pm 9.9$ & $42.5 \pm 10.0$ & $40.0 \pm 11.4$ & $0.039^{*}$ \\
Ejection fraction [\%] & $62.3 \pm 5.3$ & $60.0 \pm 4.8$ & $58.2 \pm 6.2$ & $0.000^{*}$ \\
Mass [g] & $116.1 \pm 26.2$ & $114.8 \pm 27.9$ & $112.6 \pm 27.0$ & 0.664 \\
GLS (\%) & $-21.8 \pm 4.8$ & $-20.7 \pm 2.5$ & $-19.3 \pm 2.9$ & 0.000 \\
Apical rotation [ $\left.{ }^{\circ}\right]$ & $12.5 \pm 4.5$ & $8.8 \pm 3.6$ & $6.0 \pm 3.2$ & $0.000^{* *}$ \\
Basal rotation [ ${ }^{\circ}$ ] & $-7.7 \pm 3.0$ & $-5.9 \pm 2.6$ & $-4.4 \pm 2.5$ & $0.000^{* *}$ \\
Twist [ ${ }^{\circ}$ ] & $20.0 \pm 6.4$ & $14.5 \pm 5.1$ & $9.8 \pm 4.5$ & $0.000^{* *}$ \\
Torsion [\%"cm] & $2.9 \pm 0.9$ & $2.1 \pm 0.9$ & $1.4 \pm 0.7$ & $0.000^{* *}$ \\
Time to peak apical rotation [ms] & $304.2 \pm 64.1$ & $310.1 \pm 93.8$ & $285.5 \pm 90.0$ & 0.093 \\
Time to peak basal rotation [ms] & $320.9 \pm 95.5$ & $313.0 \pm 89.5$ & $338.4 \pm 115.9$ & 0.189 \\
Time to twist [ms] & $302.8 \pm 59.2$ & $311.4 \pm 73.4$ & $307.8 \pm 82.4$ & 0.696 \\
Apical-basal rotation delay [ms] & $38.3 \pm 67.9$ & $66.7 \pm 73.9$ & $92.6 \pm 96.9$ & $0.000^{* *}$ \\
SDI [\%] & $5.4 \pm 1.6$ & $5.6 \pm 1.6$ & $5.6 \pm 1.6$ & 0.779 \\
\hline
\end{tabular}

${ }^{*} \mathrm{p}<0.05$, compared between baselines and after the fourth cycle of therapy; ${ }^{*} \mathrm{p}<0.01$, compared between baselines and after the second cycle of therapy; GLS - global longitudinal strain; SDI — systolic dyssynchrony index 


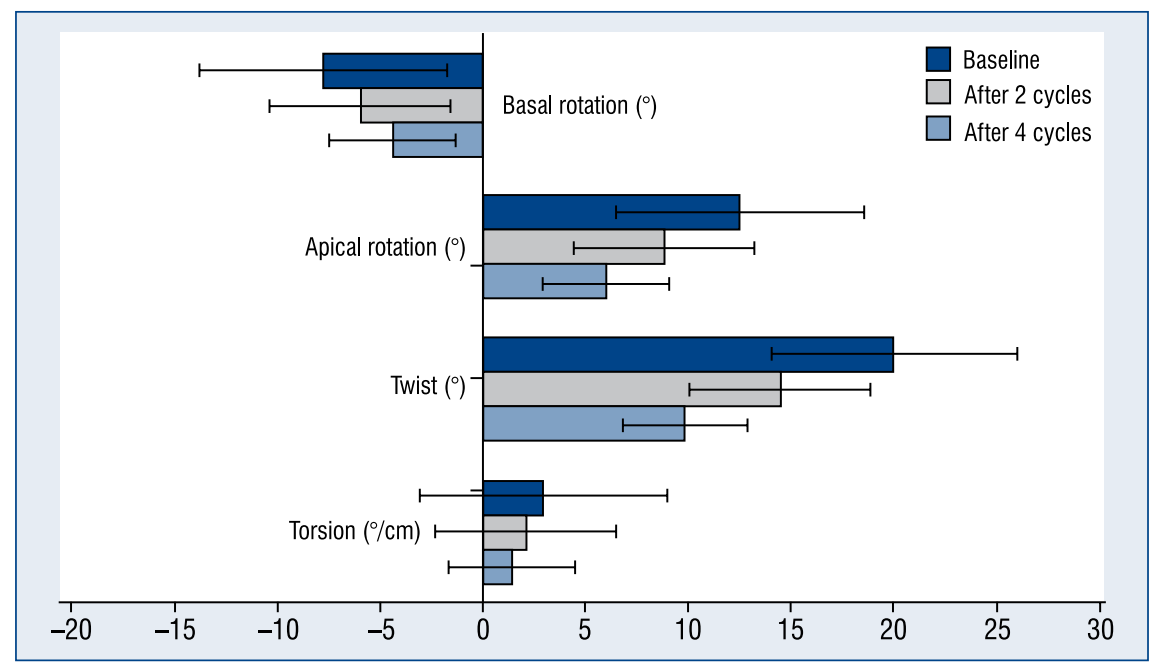

Figure 1. The variation trend of apical and basal rotation, twist as well as torsion at baseline, after 2 cycles and 4 cycles of anthracycline therapy.

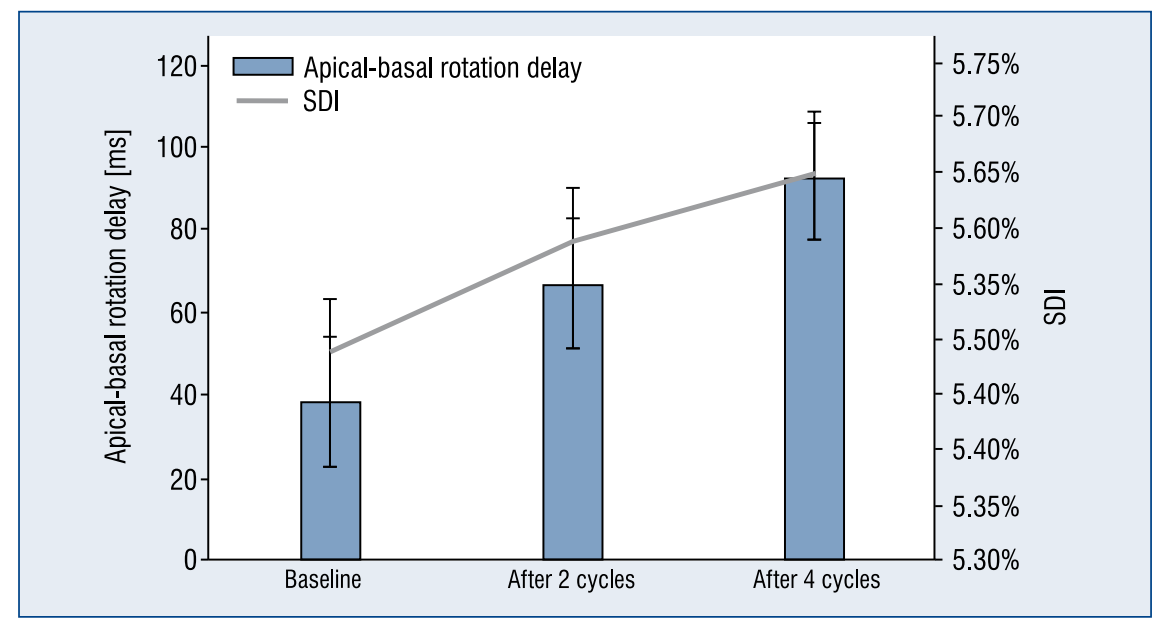

Figure 2. The difference of apical-basal rotation delay and systolic dyssynchrony index (SDI) during the entire therapy.

In systole, the base rotates in an overall clockwise direction and the apex rotates in a counterclockwise direction when viewed from apex to base, LV twist in a counterclockwise direction viewed from the apex to base. As in the Table 2, LV apical rotation, basal rotation, twist and torsion had significantly reduced after the second cycle of the regimen from $12.5 \pm 4.5^{\circ}$ to $8.8 \pm 3.6^{\circ},-7.7 \pm 3.0^{\circ}$ to $-5.9 \pm 2.6^{\circ}, 20.0 \pm 6.4^{\circ}$ to $14.5 \pm 5.1^{\circ}, 2.9 \pm 0.9^{\circ} / \mathrm{cm}$ to $2.1 \pm 0.9^{\circ} \mathrm{cm}$ (all p < 0.01). Apical-basal rotation delay was prolonged from $38.4 \pm 67.9 \mathrm{~ms}$ to $66.7 \pm 73.9 \mathrm{~ms}$ simultaneously $(\mathrm{p}<0.01)$. The trends of all these twisting variations above continued to after 4 cycles of the regimen (all $p<0.01$ ) (Figs. 1,2).
While LV SDI, time to peak apical rotation, time to peak basal rotation and time to peak twist showed no significant degradation between the pre- and posttherapies (all $\mathrm{p}>0.05$ ). LV twisting curves analyzed by $3 \mathrm{D}-\mathrm{STE}$ in a lymphoma patient before chemotherapy are shown in the Figure 3 and Figure 4 illustrating LV twisting decline curves in the same case of lymphoma after 2 cycles of treatment.

\section{Serum biochemical markers}

Serum for hs-cTnT and pro-BNP assays were collected from all subjects. Compared with the baseline, hs-cTnT was elevated significantly from $0.004 \mathrm{ng} / \mathrm{mL}$ (interquartile range: $0.003 \mathrm{ng} / \mathrm{mL}$, 


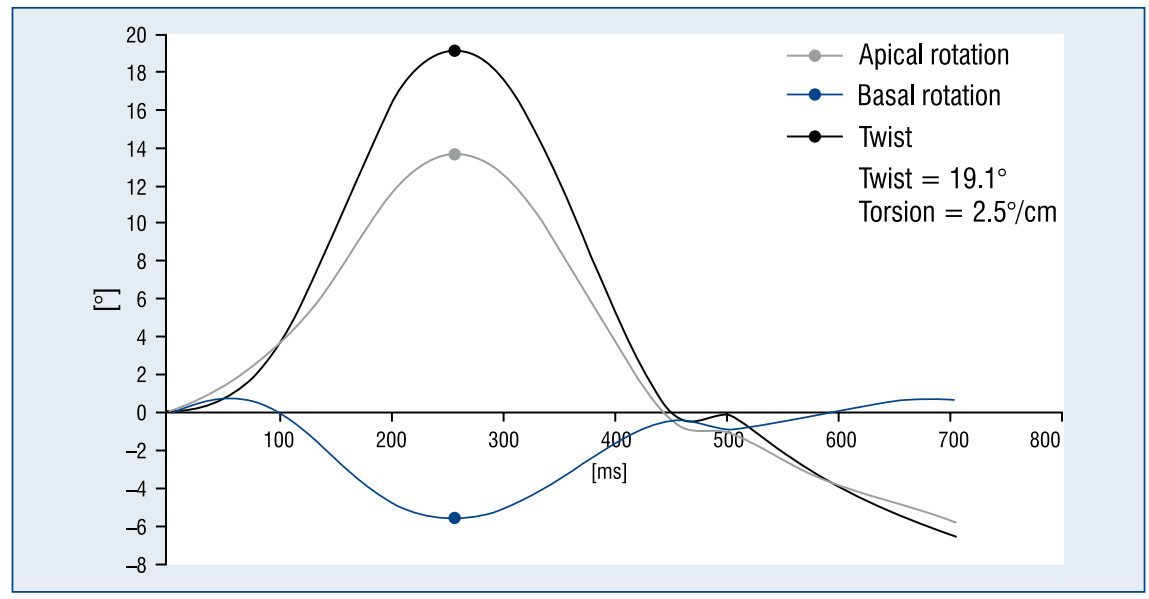

Figure 3. Example of left ventricular apical rotation, basal rotation, and twist in a lymphoma patient at baseline. Clockwise rotation as viewed from the apex was considered as negative and counterclockwise rotation as positive. The apex rotated counterclockwise, the base rotated clockwise, and the left ventricle twisted counterclockwise in systole.

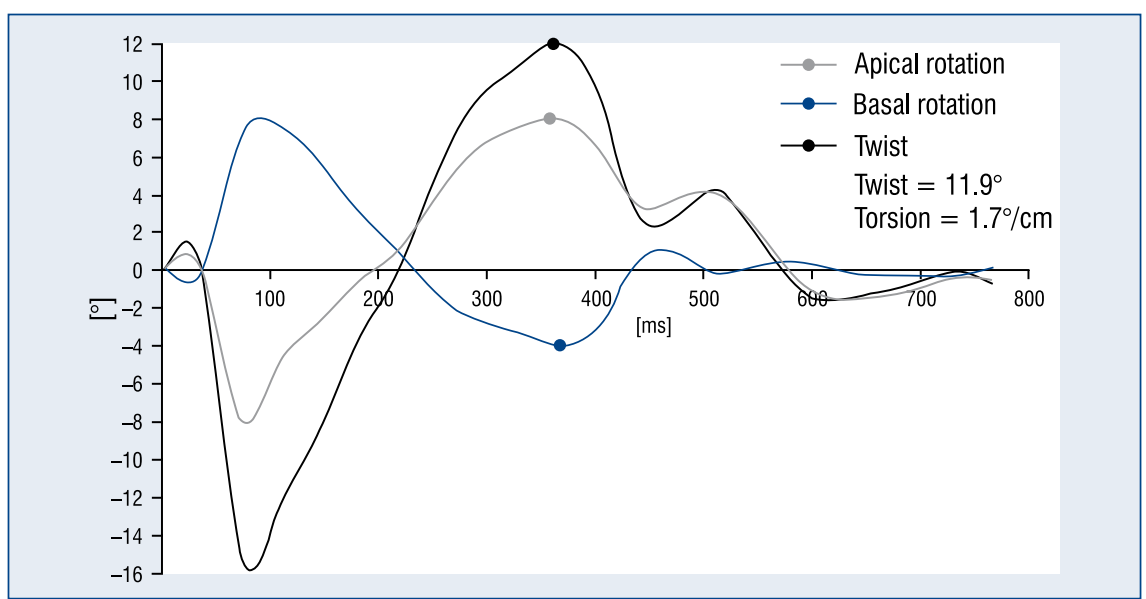

Figure 4. Example of left ventricular apical rotation, basal rotation, and twist in the same lymphoma patient after 2 cycles of anthracycline therapy. Apical rotation, basal rotation and left ventricular twist were significantly reduced, despite the same direction of apical rotation, basal rotation, and twist in the patient compared with baseline.

$0.006 \mathrm{ng} / \mathrm{mL}$ ) to $0.007 \mathrm{ng} / \mathrm{mL}$ (interquartile range: $0.004 \mathrm{ng} / \mathrm{mL}, 0.010 \mathrm{ng} / \mathrm{mL})(\mathrm{p}<0.01)$ after 2 cycles, and to $0.015 \mathrm{ng} / \mathrm{mL}$ (interquartile range: 0.011 $\mathrm{ng} / \mathrm{mL}, 0.021 \mathrm{ng} / \mathrm{mL}$ ) after 4 cycles of R-CHOP $(\mathrm{p}<0.01)$. However the mean value of pro-BNP showed no significant difference between the preand post-therapies (all $\mathrm{p}>0.05$ ).

\section{Correlations between twisting measurements and LVEF, GLS, and hs-cTnT}

The correlations between twisting parameters and LVEF, GLS and hs-cTnT are shown in the Table 4. Among these twisting parameters, torsion was most significantly correlated with LVEF $(r=0.424$, $\mathrm{p}<0.01)$, GLS $(\mathrm{r}=-0.463, \mathrm{p}<0.01)$ and hs-cTnT $(\mathrm{r}=0.506, \mathrm{p}<0.01)$.

\section{Inter- and intra-observer variation}

Inter-observer measurement showed an ICC $=0.864$ for twist, 0.925 for torsion, intra-observer measurement showed an ICC $=0.850$ for twist, 0.915 for torsion, indicating satisfactory reproductivity of primary focus indicators (Fig. 5).

\section{Discussion}

Early detection of cardiac function abnormality is of paramount importance in patients after 
Table 4. Correlations between twisting measurements and left ventricular ejection fraction (LVEF), global longitudinal strain (GLS), and high-sensitivity cardiac troponin T (hs-cTnT).

\begin{tabular}{|c|c|c|c|c|c|c|}
\hline \multirow[t]{2}{*}{ Variables } & \multicolumn{2}{|c|}{ LVEF } & \multicolumn{2}{|c|}{ GLS } & \multicolumn{2}{|c|}{ hs-cTnT } \\
\hline & $\mathbf{p}$ & $\mathbf{r}$ & $\mathbf{p}$ & $\mathbf{r}$ & $\mathbf{p}$ & $\mathbf{r}$ \\
\hline Apical rotation $\left[{ }^{\circ}\right]$ & 0.000 & $0.312^{*}$ & 0.000 & $-0.312^{*}$ & 0.000 & $0.347^{*}$ \\
\hline Basal rotation $\left[{ }^{\circ}\right]$ & 0.000 & $-0.297^{*}$ & 0.000 & $0.323^{*}$ & 0.000 & $0.364 *$ \\
\hline Twist $\left[^{\circ}\right]$ & 0.000 & $0.352^{*}$ & 0.000 & $-0.362^{*}$ & 0.000 & $0.402^{*}$ \\
\hline Torsion $[\% / \mathrm{cm}]$ & 0.000 & $0.424^{*}$ & 0.000 & $-0.463^{*}$ & 0.000 & $0.506^{*}$ \\
\hline Apical-basal rotation delay [ms] & 0.114 & - & 0.05 & - & 0.004 & $0.167^{*}$ \\
\hline
\end{tabular}

${ }^{*} p<0.01$

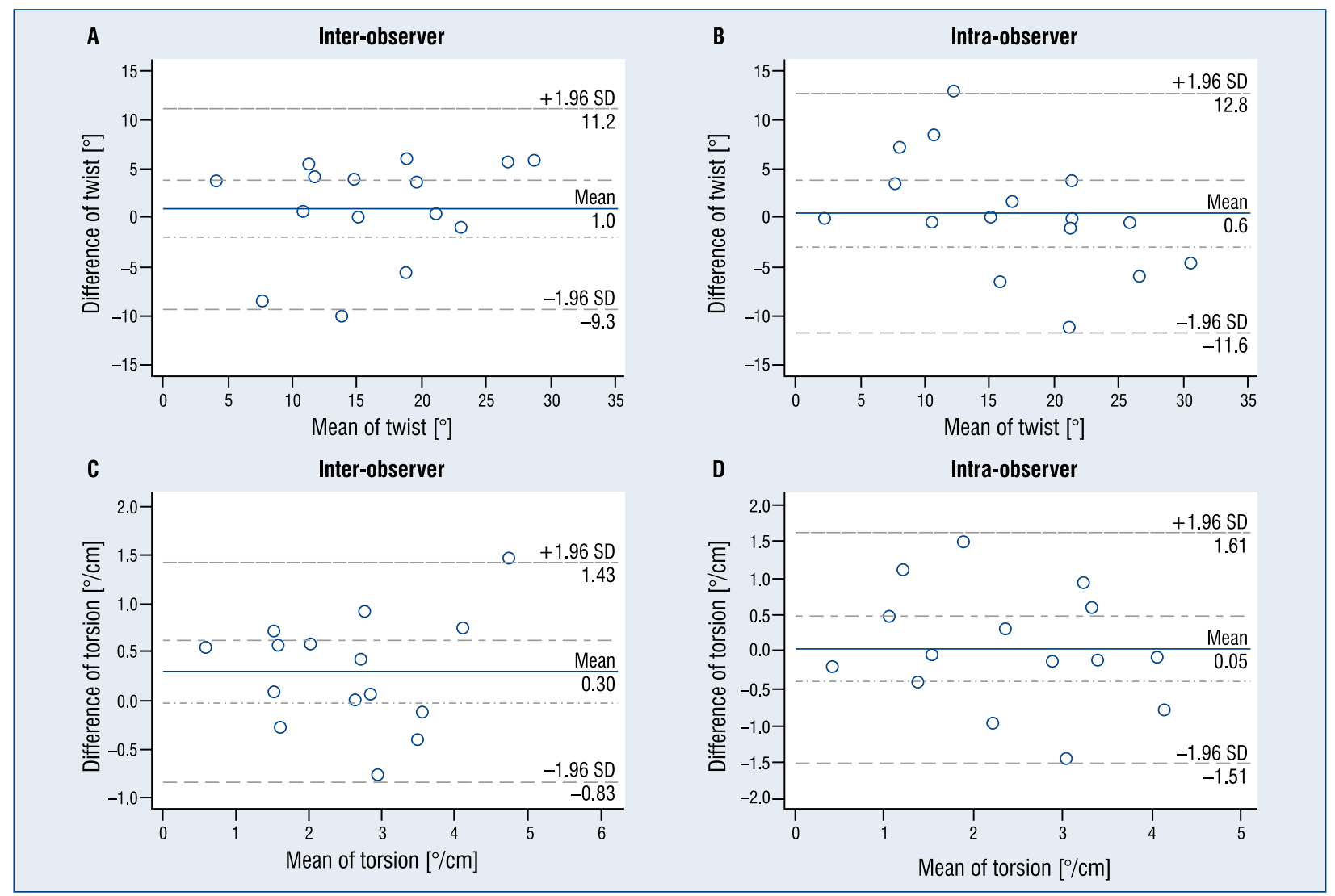

Figure 5. Bland-Altman analysis for inter-observer and intra-observer reliability for twist (A, B) and torsion (C, D); SD - standard deviation.

anthracycline therapy [12-16]. Anthracyclineinduced cardiomyocytes cell death by apoptosis and myofilament degradation may lead to impaired myocardial deformation as well as systolic dysfunction [17]. Echocardiography is the common modality for cardiac function [18-20]. 3D-STE as a novel echocardiographic technique has allowed advances in comprehensive evaluation of cardiac mechanical movement among various clinical sce- narios [21-24]. Furthermore, one of the particular advantages of 3D-STE is the improved accuracy of LV twisting measurements [25, 26]. This study showed feasibility and reproducibility of 3D-STE in assessment of early changes in LV twisting function in patients with lymphoma exposed to anthracycline.

To date, there are limited studies focusing on LV rotational function in patients associated with 
anthracycline therapy. Cheung et al. [27] reported peak LV torsion and systolic twisting velocity were significantly lower than controls in childhood cancer survivors. Similar findings were discovered by $\mathrm{Yu}$ et al. [28] which showed torsion reduced significantly in anthracycline-treated survivors of childhood cancers. In the study of Motoki et al. [29], remarkable deterioration in torsion, twisting rate were found in adult patients 1 month after chemotherapy, in the meantime, a significant negative correlation was observed between cumulative anthracycline doses and torsion. A larger cohort involving 74 adult patients revealed that LV apical rotation and twist deteriorated before LVEF decreased at 6 weeks after initiation of chemotherapy [30]. In contrast to the previous studies above, this study uses 3D-STE which detected LV twisting abnormality only after 2 cycles of anthracycline treatment. What's more, at the same time, hs-cTnT elevated significantly when compared with baseline data. The findings of reduced twist, torsion, and other rotational function parameters in the present study agree with those reported previously. It was also found that apical-basal rotation delay prolonged significantly after the second cycle of regimen, revealing obvious dyssynchrony between apical rotation and basal rotation.

Left ventricular twisting and recoil are the results of the dynamic interaction between epicardial and endocardial fiber wound oppositely and that sub-endocardial fibers are right-hand oriented and sub-epicardial fibers are left-hand oriented [31]. Because LV rotation is directly related to myocardial fiber orientation, it might be sensitive in response to various CVD which are associated with myofibrillar degradation. Depending on the extent and severity of cardiomyocyte loss as well as myocardial fibrosis induced by anthracycline [32], twisting movement and myocardial deformation may be reduced. Previous studies further demonstrated anthracycline-induced calpain-dependent titin proteolysis and necrosis in cardiomyocytes affected the fibers architecture and cardiac geometry which were verified to alter the cardiac torsion pattern [33, 34].

In the existing research, few studies have suggested that LV twisting parameters increased in given specific pathological conditions. Increased apical rotation in concentric hypertrophy and enhanced basal rotation in hypertrophic cardiomyopathy respectively were found in the study of Prinz et al. [35] and of Ahmed et al. [36], they found that $\mathrm{LV}$ torsion and twist were higher in hypertension patients. Pagourelias et al. [37] also reported that
LV twist increased in cirrhosis patients by $2 \mathrm{D}$ speckle tracking echocardiography. In these cases, $\mathrm{LV}$ systolic function is maintained with preserved or increased LVEF. Increased LVEF and LV pump function could be attributed to enhanced LV rotation movement [38, 39]. However, in anthracyclineinduced cardiomyopathy, both of the subendocardial and subepicardial helix of fibers were involved [40]. And, our study showed that LV rotational parameters reduced obviously after only 2 cycles of the therapy, and GLS and LVEF declined at the end of the therapy. This means that subclinical impairment of LV systolic function has been caused in the early stage of anthracycline therapy.

An encouraging finding of our investigation is that, reduced apical rotation, basal rotation, twist and torsion after 2 cycles of anthracycline treatment were accompanied with significant increase in hs-cTnT levels with preserved GLS as well as LVEF. While GLS is controlled predominantly by sub-endocardial fibers and it has been considered as a good marker of LV subclinical dysfunction $[41,42]$. And a relative percentage reduction of GLS of $>15 \%$ from baseline may suggest risk of cardiotoxicity during the expert consensus statement [43]. In the present study, although 3D GLS of $L V$ decreased at the end of the therapy, none of the patients reached the above stated standard. Of note, in drug-induced cardiac injury, both myocardial layers are involved inevitably [40].Thus, LV rotation could provide comprehensive information for ventricular performance. Moreover, this study suggested LV twisting parameters were more sensitive to GLS in monitoring cardiac changes after anthracycline therapy.

According to a recent expert consensus statement, a decrease in the LVEF of $>10 \%$, to a value below the lower limit of normal, is defined as cancer therapeutics-related cardiac dysfunction [43, 44]. While in this study, none of the patients developed clinical cardiotoxity as well as meeting the above standard. Tan et al. [45] reported LVEF decreased in a cohort of women with breast cancer at the end of treatment when compared with baseline (from $64 \pm 6 \%$ to $59 \pm 8 \%$ ). This result was similar to the present study, their patient EF did not decrease sufficiently to meet the diagnostic criteria by the recommended statement. Although these subjects were treated with not only anthracycline but also taxanes and trastuzumab. Clinical trials have revealed that the patients exposed to both anthracycline and trastuzumab were at greater risk for severe heart failure and reduction in LVEF than those treated with non-trastuzumab-based therapy 
[46] and some of the cardiotoxicity may also be related to the taxane [47]. It appears therefore, to be quite difficult to achieve the guideline standard at an early stage after anthracycline therapy, despite modest cardiac injury having been induced. A large study by Khan et al. [48], involving 1204 patients, showed $10.2 \%(\mathrm{n}=123)$ patients occurred cardiotoxicity which was defined as a reduction in LVEF $\geq 10 \%$ absolute points from baseline and LVEF $<55 \%$. Meanwhile, another study with 75 patients reported that $18.67 \%$ patients developed an asymptomatic reduction of the LVEF of $\geq 10 \%$ after epirubicin therapy [49]. The present results appeared to be discordant with these two studies, however, their patients had received anthracycline $>300 \mathrm{mg} / \mathrm{m}^{2}$ which were higher than our cumulative dose. Meanwhile, it was believed that different patients, follow-up time and chemotherapy regimen may account for the various results, LVEF itself may be not efficient enough to identify subtle myocardial systolic performance when compared with other novel echocardiographic indexes.

Cardiac biomarkers including natriuretic peptides and troponins have been identified as useful tools for assessing cancer therapeutics-related cardiac dysfunction [50-52]. However, conclusive data are needed to establish whether biomarkers reliably predict clinically relevant late consequences of cancer treatment when the patients combined with other CVD [43]. The determination of the optimal timing and assay platform of natriuretic peptides and troponins assessment remains in question. In this study, measurement of pro-BNP and hs-cTnT in lymphoma patients whom were free of noncancerous CVD for every two cycles of treatment was conducted. Pro-BNP presented no significant difference between the pre- and post-therapies, while hs-cTnT levels elevated markedly after the second cycle of R-CHOP that was inconsistent with twisting variations. Moreover, the correlation analysis results between hs-cTnT and twisting parameters indicated were satisfactory. The integrated approach combining hs-cTnT and rotational parameters hence may provide incremental value in monitoring prior cardiac damage induced by anthracycline treatment.

\section{Limitations of the study}

Several limitations to this study warrant comments. Firstly, although this study enrolled a large cohort which included more than 100 cancer patients, it is a single center study and its reproduction in other centers or by multicenter studies could support its validity. Secondly, information was not provided on the prediction value of LV rotational indexes in prognostication. Finally, it would have been ideal to add normal control group in this study. Patient parameters were analyzed only the by own control comparison.

\section{Conclusions}

Small changes in myocardial twisting after anthracycline-embedded chemotherapy can be revealed by using 3D-STE. LV twisting appears to be a promising measure for quantitative detection of subclinical systolic dysfunction induced by anthracycline.

\section{Acknowledgements}

This work was supported by the National Nature Science Foundation of China (NO 81201095) and Excellent backbone plan of Zhongshan Hospital, Fudan University (NO 2015ZSYXGG04).

\section{Conflict of interest: None declared}

\section{References}

1. DeSantis CE, Lin CC, Mariotto AB, et al. Cancer treatment and survivorship statistics, 2014. CA Cancer J Clin. 2014; 64(4): $252-$ 271, doi: 10.3322/caac.21235, indexed in Pubmed: 24890451.

2. Haddy N, Mousannif A, Tukenova M, et al. Role of cancer treatment in long-term overall and cardiovascular mortality after childhood cancer. J Clin Oncol. 2010; 28(8): 1308-1315, doi: 10.1200/JCO.2008.20.2267, indexed in Pubmed: 20142603.

3. Mertens AC, Liu Qi, Neglia JP, et al. Cause-specific late mortality among 5-year survivors of childhood cancer: the Childhood Cancer Survivor Study. J Natl Cancer Inst. 2008; 100(19): 1368-1379, doi: 10.1093/jnci/djn310, indexed in Pubmed: 18812549.

4. Notomi Y, Lysyansky P, Setser RM, et al. Measurement of ventricular torsion by two-dimensional ultrasound speckle tracking imaging. J Am Coll Cardiol. 2005; 45(12): 2034-2041, doi: 10.1016/j.jacc.2005.02.082, indexed in Pubmed: 15963406.

5. Rüssel IK, Götte MJW, Bronzwaer JG, et al. Left ventricular torsion: an expanding role in the analysis of myocardial dysfunction. JACC Cardiovasc Imaging. 2009; 2(5): 648-655, doi: 10.1016/j. jcmg.2009.03.001, indexed in Pubmed: 19442954.

6. Cutrì E, Serrani M, Bagnoli P, et al. The cardiac torsion as a sensitive index of heart pathology: A model study. J Mech Behav Biomed Mater. 2015; 55: 104-119, doi: 10.1016/j. jmbbm.2015.10.009, indexed in Pubmed: 26580023.

7. Kowallick JT, Lamata P, Hussain ST, et al. Quantification of left ventricular torsion and diastolic recoil using cardiovascular magnetic resonance myocardial feature tracking. PLoS One. 2014; 9(10): e109164, doi: 10.1371/journal.pone.0109164, indexed in Pubmed: 25285656.

8. Zhong SW, Zhang YQ, Chen LJ, et al. Ventricular twisting and dyssynchrony in children with single left ventricle using threedimensional speckle tracking imaging after the fontan operation. Echocardiography. 2016; 33(4): 606-617, doi: 10.1111/ /echo.13103, indexed in Pubmed: 26606913. 
9. Zhou X, Thavendiranathan P, Chen Y, et al. Feasibility of automated three-dimensional rotational mechanics by real-time volume transthoracic echocardiography: Preliminary accuracy and reproducibility data compared with cardiovascular magnetic resonance. J Am Soc Echocardiogr. 2016; 29(1): 62-73, doi: 10.1016/j. echo.2015.07.027, indexed in Pubmed: 26363710.

10. Lang RM, Bierig M, Devereux RB, et al. Recommendations for chamber quantification. Eur J Echocardiogr. 2006; 28(12): 79-108, doi: 10.1016/j.euje.2005.12.014.

11. Freund Y, Chenevier-Gobeaux C, Bonnet P, et al. High-sensitivity versus conventional troponin in the emergency department for the diagnosis of acute myocardial infarction. Crit Care. 2011; 15(3): R147, doi: 10.1186/cc10270, indexed in Pubmed: 21663627.

12. Cardinale D, Colombo A, Bacchiani G, et al. Early detection of anthracycline cardiotoxicity and improvement with heart failure therapy. Circulation. 2015; 131(22): 1981-1988, doi: 10.1161/CIRCULATIONAHA.114.013777, indexed in Pubmed: 25948538.

13. van der Pal HJ, van Dalen EC, van Delden E, et al. High risk of symptomatic cardiac events in childhood cancer survivors. J Clin Oncol. 2012; 30(13): 1429-1437, doi: 10.1200/JCO.2010.33.4730, indexed in Pubmed: 22473161.

14. van der Pal HJ, van Dalen EC, Hauptmann M, et al. Cardiac function in 5-year survivors of childhood cancer: a long-term follow-up study. Arch Intern Med. 2010; 170(14): 1247-1255, doi: 10.1001/ /archinternmed.2010.233, indexed in Pubmed: 20660845.

15. Gianni L, Herman EH, Lipshultz SE, et al. Anthracycline cardiotoxicity: from bench to bedside. J Clin Oncol. 2008; 26(22): 3777-3784, doi: 10.1200/JCO.2007.14.9401, indexed in Pubmed: 18669466.

16. Rickard J, Kumbhani DJ, Baranowski B, et al. Usefulness of cardiac resynchronization therapy in patients with adriamycin-induced cardiomyopathy. Am J Cardiol. 2010; 105(4): 522-526, doi: 10.1016/j.amjcard.2009.10.024, indexed in Pubmed: 20152248.

17. Chen B, Peng X, Pentassuglia L, et al. Molecular and cellular mechanisms of anthracycline cardiotoxicity. Cardiovasc Toxicol. 2007; 7(2): 114-121, doi: 10.1007/s12012-007-0005-5, indexed in Pubmed: 17652815.

18. Mast TP, Teske AJ, Doevendans PA, et al. Current and future role of echocardiography in arrhythmogenic right ventricular dysplasia/cardiomyopathy. Cardiol J, 2015; 22(4): 362-374, doi: 10.5603/CJ.a2015.0018.

19. Lipiec P, Wejner-Mik P, Wdowiak-Okrojek K, et al. Fusion of morphological data obtained by coronary computed tomography angiography with quantitative echocardiographic data on regional myocardial function. Cardiol J, 2016; 23(3): 264-269, doi: 10.5603/CJ.a2016.0015.

20. Kang Y, Cheng L, Cui J, et al. A new score system for predicting response to cardiac resynchronization therapy. Cardiol J, 2015; 22(2): 179-187, doi: 10.5603/CJ.a2014.0089.

21. Jenkins C, Chan J, Hanekom L, et al. Accuracy and feasibility of online 3-dimensional echocardiography for measurement of left ventricular parameters. J Am Soc Echocardiogr. 2006; 19(9): 1119-1128, doi: 10.1016/j.echo.2006.04.002, indexed in Pubmed: 16950466.

22. Burri MV, Gupta D, Kerber RE, et al. Review of novel clinical applications of advanced, real-time, 3-dimensional echocardiography. Transl Res. 2012; 159(3): 149-164, doi: 10.1016/j. trsl.2011.12.008, indexed in Pubmed: 22340764.
23. Vamvakidou A, Gurunathan S, Senior R. Novel techniques in stress echocardiography: A focus on the advantages and disadvantages. Expert Rev Cardiovasc Ther. 2016; 14(4): 477-494, doi: 10.1586/14779072.2016.1135054, indexed in Pubmed: 26686698.

24. Luis SA, Yamada A, Khandheria BK, et al. Use of three-dimensional speckle-tracking echocardiography for quantitative assessment of global left ventricular function: a comparative study to three-dimensional echocardiography. J Am Soc Echocardiogr. 2014; 27(3): 285-291, doi: 10.1016/j.echo.2013.11.002, indexed in Pubmed: 24325960.

25. Jasaityte R, Heyde B, D'hooge J. Current state of three-dimensional myocardial strain estimation using echocardiography. J Am Soc Echocardiogr. 2013; 26(1): 15-28, doi: 10.1016/j. echo.2012.10.005, indexed in Pubmed: 23149303.

26. Seo Y, Ishizu T, Aonuma K. Current status of 3-dimensional speckle tracking echocardiography: A review from our experiences. J Cardiovasc Ultrasound. 2014; 22(2): 49-57, doi: 10.4250/ /jcu.2014.22.2.49, indexed in Pubmed: 25031794.

27. Cheung Yf, Li Sn, Chan GCF, et al. Left ventricular twisting and untwisting motion in childhood cancer survivors. Echocardiography. 2011; 28(7): 738-745, doi: 10.1111/j.15408175.2011.01429.x, indexed in Pubmed: 21615484.

28. Yu Hk, Yu W, Cheuk DKL, et al. New three-dimensional speckletracking echocardiography identifies global impairment of left ventricular mechanics with a high sensitivity in childhood cancer survivors. J Am Soc Echocardiogr. 2013; 26(8): 846-852, doi: 10.1016/j.echo.2013.04.018, indexed in Pubmed: 23727115.

29. Motoki H, Koyama J, Nakazawa H, et al. Torsion analysis in the early detection of anthracycline-mediated cardiomyopathy. Eur Heart J Cardiovasc Imaging. 2012; 13(1): 95-103, doi: 10.1093/ /ejechocard/jer172, indexed in Pubmed: 21926409.

30. Mornoş C, Petrescu L. Early detection of anthracycline-mediated cardiotoxicity: the value of considering both global longitudinal left ventricular strain and twist. Can J Physiol Pharmacol. 2013; 91(8): 601-607, doi: 10.1139/cjpp-2012-0398, indexed in Pubmed: 23889668.

31. Sengupta PP, Tajik AJ, Chandrasekaran K, et al. Twist mechanics of the left ventricle: principles and application. JACC Cardiovasc Imaging. 2008; 1(3): 366-376, doi: 10.1016/j.jcmg.2008.02.006, indexed in Pubmed: 19356451.

32. Yamaoka M, Yamaguchi S, Suzuki T, et al. Apoptosis in rat cardiac myocytes induced by Fas ligand: priming for Fas-mediated apoptosis with doxorubicin. J Mol Cell Cardiol. 2000; 32(6): 881-889, doi: 10.1006/jmcc.2000.1132, indexed in Pubmed: 10888243.

33. Lim CC, Zuppinger C, Guo X, et al. Anthracyclines induce calpain-dependent titin proteolysis and necrosis in cardiomyocytes. J Biol Chem. 2004; 279(9): 8290-8299, doi: 10.1074/jbc. M308033200, indexed in Pubmed: 14676206.

34. Bell SP, Nyland L, Tischler MD, et al. Alterations in the determinants of diastolic suction during pacing tachycardia. Circ Res. 2000; 87(3): 235-240, indexed in Pubmed: 10926875.

35. Prinz C, Faber L, Horstkotte D, et al. Evaluation of left ventricular torsion in children with hypertrophic cardiomyopathy. Cardiol Young. 2014; 24(2): 245-252, doi: 10.1017/S104795111300005X, indexed in Pubmed: 23388176.

36. Ahmed MI, Desai RV, Gaddam KK, et al. Relation of torsion and myocardial strains to LV ejection fraction in hypertension. JACC Cardiovasc Imaging. 2012; 5(3): 273-281, doi: 10.1016/j. jcmg.2011.11.013, indexed in Pubmed: 22421172.

37. Pagourelias ED, Sotiriou P, Papadopoulos CE, et al. Left ventricular myocardial mechanics in cirrhosis: A speckle tracking echo- 
cardiographic study. Echocardiography. 2016; 33(2): 223-232, doi: 10.1111/echo.13010, indexed in Pubmed: 26174780.

38. Di Maria MV, Caracciolo G, Prashker S, et al. Left ventricular rotational mechanics before and after exercise in children. J Am Soc Echocardiogr. 2014; 27(12): 1336-1343, doi: 10.1016/j. echo.2014.07.016, indexed in Pubmed: 25204858.

39. Aksakal E, Kurt M, Oztürk ME, et al. The effect of incremental endurance exercise training on left ventricular mechanics: a prospective observational deformation imaging study. Anadolu Kardiyol Derg. 2013; 13(5): 432-438, doi: 10.5152/akd.2013.137, indexed in Pubmed: 23665984.

40. Sengupta PP, Narula J. Reclassifying heart failure: predominantly subendocardial, subepicardial, and transmural. Heart Fail Clin. 2008; 4(3): 379-382, doi: 10.1016/j.hfc.2008.03.013, indexed in Pubmed: 18598989.

41. Armstrong GT, Joshi VM, Ness KK, et al. Comprehensive echocardiographic detection of treatment-related cardiac dysfunction in adult survivors of childhood cancer: Results from the St. Jude Lifetime Cohort Study. J Am Coll Cardiol. 2015; 65(23): 2511-2522, doi: 10.1016/j.jacc.2015.04.013, indexed in Pubmed: 26065990.

42. Thavendiranathan P, Poulin F, Lim KD, et al. Use of myocardial strain imaging by echocardiography for the early detection of cardiotoxicity in patients during and after cancer chemotherapy: a systematic review. J Am Coll Cardiol. 2014; 63(25 Pt A): 2751-2768, doi: 10.1016/j.jacc.2014.01.073, indexed in Pubmed: 24703918.

43. Zamorano JL, Lancellotti P, Rodriguez Muñoz D, et al. Authors/ Task Force Members, ESC Committee for Practice Guidelines (CPG):. 2016 ESC Position Paper on cancer treatments and cardiovascular toxicity developed under the auspices of the ESC Committee for Practice Guidelines: The Task Force for cancer treatments and cardiovascular toxicity of the European Society of Cardiology (ESC). Eur Heart J. 2016; 37(36): 27682801, doi: 10.1093/eurheartj/ehw211, indexed in Pubmed: 27567406.

44. Lang RM, Badano LP, Mor-Avi V, et al. Recommendations for cardiac chamber quantification by echocardiography in adults: an update from the American Society of Echocardiography and the European Association of Cardiovascular Imaging.
J Am Soc Echocardiogr. 2015; 28(1): 1-39.e14, doi: 10.1016/j. echo.2014.10.003, indexed in Pubmed: 25559473.

45. Tan TC, Bouras S, Sawaya H, et al. Time trends of left ventricular ejection fraction and myocardial deformation indices in a cohort of women with breast cancer treated with anthracyclines, taxanes, and trastuzumab. J Am Soc Echocardiogr. 2015; 28(5): 509-514, doi: 10.1016/j.echo.2015.02.001, indexed in Pubmed: 25772019.

46. Moja L, Tagliabue L, Balduzzi S, et al. Trastuzumab containing regimens for early breast cancer. Cochrane Database Syst Rev. 2012(4): CD006243, doi: 10.1002/14651858.CD006243.pub2, indexed in Pubmed: 22513938.

47. Guarneri V, Lenihan DJ, Valero V, et al. Long-term cardiac tolerability of trastuzumab in metastatic breast cancer: the M.D. Anderson Cancer Center experience. J Clin Oncol. 2006; 24(25): 4107-4115, doi: 10.1200/JC0.2005.04.9551, indexed in Pubmed: 16908934.

48. Khan AA, Ashraf A, Singh R, et al. Incidence, time of occurrence and response to heart failure therapy in patients with anthracycline cardiotoxicity. Intern Med J. 2017; 47(1): 104-109, doi: 10.1111/imj.13305, indexed in Pubmed: 27800661.

49. Kang Yu, Xu X, Cheng L, et al. Two-dimensional speckle tracking echocardiography combined with high-sensitive cardiac troponin $\mathrm{T}$ in early detection and prediction of cardiotoxicity during epirubicine-based chemotherapy. Eur J Heart Fail. 2014; 16(3): 300-308, doi: 10.1002/ejhf.8, indexed in Pubmed: 24464946.

50. Sanchis J, Bardají A, Bosch X, et al. N-terminal pro-brain natriuretic peptide and high-sensitivity troponin in the evaluation of acute chest pain of uncertain etiology. A PITAGORAS substudy. Rev Esp Cardiol (Engl Ed). 2013; 66(7): 532-538, doi: 10.1016/j. rec.2012.11.004, indexed in Pubmed: 24776201.

51. Blaes AH, Rehman A, Vock DM, et al. Utility of high-sensitivity cardiac troponin $\mathrm{T}$ in patients receiving anthracycline chemotherapy. Vasc Health Risk Manag. 2015; 11: 591-594, doi: 10.2147/VHRM.S89842, indexed in Pubmed: 26648730.

52. Zidan A, Sherief LM, El-sheikh A, et al. NT-proBNP as early marker of subclinical late cardiotoxicity after doxorubicin therapy and mediastinal irradiation in childhood cancer survivors. Dis Markers. 2015; 2015: 513219, doi: 10.1155/2015/513219, indexed in Pubmed: 25960594. 